\title{
Evaluation of Steroid Sex Hormone Levels in Cockatiels During Their Reproductive Cycle
}

\author{
Hsin-Tai Hong ${ }^{1} \&$ Chean-Ping $\mathrm{Wu}^{1}$ \\ ${ }^{1}$ Department of Animal Science, National Chiayi University, Chiayi, Taiwan \\ Correspondence: Chean-Ping Wu, Department of Animal Science, National Chiayi University, 300 University \\ Road, Chiayi 60004, Taiwan. Tel: 886-5271-7532. Fax: 886-5271-7527. E-mail: wcp@mail.ncyu.edu.tw
}

Received: November 26, 2018

Accepted: December 26, 2018

Online Published: February 15, 2019

doi:10.5539/jas.v11n3p172

URL: https://doi.org/10.5539/jas.v11n3p172

\begin{abstract}
Sexual steroid hormone concentrations differ with respect to the different stages of animal life. For example, hormone concentrations in the reproductive stage show a periodic variation. Studies on variations in sexual hormones in parrots using non-invasive methods as well as the effect of nest-boxes in parrots are less. This study aimed to detect the levels of fecal steroid hormones during the cockatiel reproductive cycle and to investigate the effect of nest-box placement on reproduction. There were 24 mating pairs of cockatiels, and the study was conducted over 13 months. Based on their reproductive status, individual birds were divided into pre-reproduction (PR), incubation (IN), and parent period (PA) in the pre-test; then, the nest box was either temporarily removed for 20 days (RN) or continuously provided (pre-reproduction, PR). The estrogen concentrations measured in the last stage of the PR period and the initial stage of the IN period were significantly higher than those measured in the PA stage, and there were no significant differences in testosterone and progesterone concentrations throughout the reproductive cycle. The nest-box treatments (NB) indicated that eggs were laid 6-13 days after the new box was hung; however, in the pre-test, there was an interval of 30-40 days before the next mating pairs started the next reproductive stage or no reproduction. Estrogen concentrations in $\mathrm{PR}$ and testosterone concentrations in $\mathrm{RN}$ were significantly higher than those in the other periods during the nest-box treatments. In conclusion, estrogen showed the most significant change among the fecal steroid hormones measured. Nest boxes serve as a positive stimulus for parrot reproduction management and can help non-seasonal reproductive parrots to rapidly enter the next breeding cycle.
\end{abstract}

\section{Introduction}

Cockatiels (Nymphicus hollandicus) are granivorous, sexually dimorphic parrots, native to the arid grassland and savannah regions of central Australia (Shields, Yamamoto, \& Millam, 1989; Martin \& Millam, 1995). They are monogamous birds, in which both parents contribute to incubation and the feeding of nestlings, of which there are often three to six per clutch (Forshaw \& Cooper, 1981). In southern Australia, the wild cockatiel populations breed seasonally, but are more or less dependent on rainfall further north. In Taiwan, which lies in a temperate zone area, cockatiels are non-seasonal breeders, breeding both in and out of season.

Nests have been regarded as a prerequisite for ovarian development, ovulation, and laying in budgerigars (Melopsittacus undulatus) (Hutchison, 1974; Putnam \& Hinde, 1973) and canaries (Serinus canarius) (Shields et al., 1989; Hinde, 1965; Hinde \& Steel, 1978). Previous studies have defined the presentation, material, and shape of preferred nest boxes for these species (Shields et al., 1989; Clark \& Mason, 1985; Appleby \& McRae, 1986). These studies indicate that provision of a nest box might be an important environmental stimulus to induce reproductive development (Martin \& Millam, 1995).

Monitoring the concentrations of hormones can provide insight into the mechanistic aspects of bird mating behavior. Hormone levels are associated with sexual, reproductive, courtship, parental, aggressive, and feeding behaviors, and many studies have revealed seasonal variation in the steroid sex hormones of avian species (Silver, Reboulleau, Lehrman, \& Feder, 1974; Schumacher \& Balthazart, 1983; Williams, 1992; Kotrschal, Dittami, Hirschenhauser, Moestl, \& Peczely, 2000). Avian steroid sex hormones can be analyzed using a number of different source materials, including plasma, feces, urine, and feathers. Collecting and analyzing animal feces has become an effective non-invasive approach for the study of animal endocrine status and stress physiology (Whitten, Brockman, \& Stavisky, 1998), and an increasing number of studies are using fecal steroid 
measurements to assess reproduction, stress, and aggression in populations of animals (Khan, Altmann, Isani, \& $\mathrm{Yu}, 2002 \mathrm{a})$. In the present study, we used feces to assay hormone levels in relation to cockatiel mating and reproductive behavior, as described previously (Whitten et al., 1998; Khan, Altmann, Isani, \& Yu, 2002b; Goymann, 2005).

Research on the relationship between nest box use and avian endocrine responses has, to date, tended to be relatively limited. For breeders of artificial breeds, the re-set of a nest-box can be used to regulate the reproduction status of parrots that are unable to reproduce for a long time period, providing more economic benefits. Furthermore, parrots play an important role in agricultural ecology and contribute to ecological balance. Our research is beneficial for the reproduction of parrots. Most captive avian species can rapidly enter the next reproductive cycle and produce more offspring, leading to the perpetuation of some avian species. Success in artificial propagation in avians can reduce the pressure of hunting for the wild animal. In this study, by altering the availability of nest boxes, we were able to observe the ability of cockatiels to regulate physiological steroid hormone levels. We speculated that the replacement of nest boxes would stimulate cockatiels to rapidly enter the next reproductive cycle. The objectives of this study were to (1) observe the cockatiel reproductive cycle, (2) assay the levels of steroid sex hormones in fecal samples from mating pairs of cockatiels, and (3) study the effect of nest box replacement on cockatiel reproduction.

\section{Methods}

\subsection{Animal Maintenance}

Twenty-four pairs of cockatiels (Nymphicus hollandicus) were obtained from bird shops in Chiayi, Taiwan. All the birds used in this study were born between April and June 2011, and fecal samples were collected when the birds were 11 months old, an age at which these birds are sufficiently mature to breed. Pairs of cockatiels were housed in cages that were approximately $132,000 \mathrm{~cm}^{3}(40 \mathrm{~cm} \times 55 \mathrm{~cm} \times 60 \mathrm{~cm})$ in size, within light-controlled rooms with a photoperiod of $10 \mathrm{~h}$ light: $14 \mathrm{~h}$ dark, at the National Chiayi University Animal Research Farm. Each cage contained wooden perches. A commercial complex cereal grain diet (Extra, Taiwan) and water were continuously provided. The average weight of the cockatiels was $102.4 \pm 13.5 \mathrm{~g}$.

\subsection{Reproductive Cycles}

Reproductive cycles were divided into three periods: pre-reproduction (PR), incubation (IN), and parental (PA). The PR period was defined as the mean time before cockatiels began to lay eggs, the IN period was when the cockatiels laid their first egg and incubated their eggs until nestlings hatched, and the PA period was when parents began to feed their nestlings. The duration of the three periods was 40, 19, and 20 days, respectively. The reproductive cycles could either be complete (including PR, IN, and PA periods) or incomplete (including only PR and IN periods).

Nest behavior is a necessary element of cockatiel reproduction. Birds that are captive-bred typically lay and incubate their eggs in nest boxes. We determined that the cockatiels that laid eggs and successfully hatched nestlings continued to do so when provided withe nest boxes. The cockatiel pairs ( 24 pairs) were divided into two groups according to their reproductive status. In one group, we re-set the nest box (NB group), whereby the box, which was external and could be easily dismantled, was removed and a new nest box was rehung after an interval of 20 days, and the dung tray of which was regularly cleaned before feeding. In the other group, the original nest box was maintained (CON group) and regularly cleaned. The NB group was further divided into the period of nest box removal (RN period, 20 days), pre-reproduction period (PR, hanging of the new nest box, 6-13 days), incubation period (IN, 19 days), and parental period (PA, 20 days). The samples of droppings produced during each period were pooled (RN1-5, PR1-7, IN1-5, and PA1-4) for steroid hormone analysis. Each period was subdivided into different stages to determine the fluctuation in steroid hormones.

\subsection{Dropping Collection, Storage, and Hormone Extraction}

For validation of assays, each morning, we collected all the fresh droppings produced by the captive cockatiels in each cage and placed these into clear tubes. Prior to feeding, we renewed the dung trays in each cage, and collected the droppings deposited within 1-1.5 hours after feeding to ensure freshness. Approximately one to two tubes of droppings were collected from the cages, each of the latter of which was considered as an experimental unit. The tubes were stored frozen at $-80^{\circ} \mathrm{C}$ until the samples were used for steroid hormone extraction. We used a fecal steroid hormone extraction method that has previously been described by (Staley, Blanco, Dufty, Wildt, \& Monfort, 2007) with minor modifications. For extraction, feces (0.05-0.08 g) were suspended in 1.5-2 mL of $80 \%$ ethanol, vortexed, incubated in a water bath at $55^{\circ} \mathrm{C}$ for $20 \mathrm{~min}$, and then centrifuged at $500-2,000 \times g$ for 15 min (the $g$-value being dependent on the amount of solid droppings). The supernatant was then decanted into 
clean tubes. This step was repeated three times using the original sample to maximize the amounts of fecal steroid sex hormones that could be extracted. The final extract was re-suspended in $1 \mathrm{~mL}$ methanol, vortexed and sonicated, and then diluted (1:50-1:500 for fecal estrogen metabolites (fEM); 1:40-1:250 for fecal progesterone metabolites (fPM); 1:8-1:64 for fecal androgen metabolites (fAM)) in assay buffer (for fA and fE metabolite extracts: steroid diluent, for fPM extracts: phosphate buffer). All the tubes were stored at $-80{ }^{\circ} \mathrm{C}$ until used for assay of the steroid sex hormones in fecal samples. The hormone recovery procedure conducted in the study has previously been described by Wasser, Monfort, Southers, and Wildt (1994), and Khan et al. (2002). Estrogen, testosterone, and progesterone (Sigma-Aldrich Co., LLC., USA) were prepared by dissolving in $80 \%$ methanol to give $3000 \mathrm{pg} / \mathrm{mL}$ solutions. To two tubes, we added $1 \mathrm{~mL}$ of one of the aforementioned hormone solutions (estrogen, testosterone, and progesterone), giving six tubes in total, and a further two tubes for each hormone were left empty as controls. All the tubes were stored frozen at $-80{ }^{\circ} \mathrm{C}$. From randomly selected dropping samples, we took 10-mg amounts and transferred these to each of the 12 tubes, followed by the addition of 500 $\mu \mathrm{L} 80 \%$ ethanol. The droppings and ethanol in the tubes were mixed for $20 \mathrm{~min}$ (at $50{ }^{\circ} \mathrm{C}$ ). Thereafter, all the tubes were centrifuged at $500-2,000 \times g$ for $15 \mathrm{~min}$ (the $g$-value being dependent on the amount of solid droppings). The supernatant was then decanted into clean tubes. This step was repeated three times on the original sample to maximize the amount of steroid sex hormone that could be extracted.

\subsection{Fecal Steroid Sex Hormone ELISA Assays}

The concentrations of steroid sex hormones (estrogen, testosterone, and progesterone) in fecal samples were determined from duplicate tubes with enzyme-linked immunoassay (ELISA), using a 17ß-Estradiol high sensitivity ELISA kit, a Testosterone high sensitivity ELISA kit, and a Progesterone ELISA kit (Enzo Life Sciences Inc., Farmingdale, NY, USA), respectively, following the manufacturer's instructions.

\subsection{Statistical Analysis}

We independently analyzed the assay data for steroid sex hormones in the fecal samples collected from 24 pairs of cockatiels. The statistical analysis used in this study was based on generalized linear model (GLMs; SAS 9.1) that has previously been described by Blas, Lopez, Tanferna, Sergio, and Hiraldo (2010) with differences considered significant at $\mathrm{P}<0.05$.

\section{Results and Discussion}

\subsection{Recovery Rate of Fecal Steroid Hormone Extracts}

The recovery rates for fecal estrogen, testosterone, and progesterone were $84.3 \%(n=8), 85 \%(n=8)$, and $82.9 \%$ $(\mathrm{n}=8)$, respectively (Table 1$)$. These recovery rates are similar to the $81.95 \%$ reported previously for fecal corticosterone in collared scops owls (Lin, 2008).

Table 1 . The extract recovery rates of fecal steroid sex hormones in cockatiels

\begin{tabular}{llll}
\hline & $3000 \mathrm{pg} / \mathrm{mL}+$ dropping hormone $(\mathrm{pg} / \mathrm{mL})$ & Dropping hormone $(\mathrm{pg} / \mathrm{mL})$ & Recovery rate $(\%)$ \\
\hline Estrogen & $5502.13 \pm 138.42$ & $2972.84 \pm 231.90$ & 84.3 \\
Testosterone & $9729.17 \pm 241.76$ & $7179.47 \pm 151.57$ & 85.0 \\
Progesterone & $6552.38 \pm 225.91$ & $4065.78 \pm 222.46$ & 82.9
\end{tabular}

Note. Recovery $=[(3000 \mathrm{pg} / \mathrm{mL}$ Hormone standard + Fecal hormone $)-$ Fecal hormone concentration $] / 3000$ $\mathrm{pg} / \mathrm{mL}$ Hormone standard $\times 100 \%$.

\subsection{Fecal Steroid Hormone Levels Change After Mating in Cockatiels}

The reproductive cycle after cockatiel mating and the curve of steroid hormone levels in cockatiel feces are shown in Figure 1. At the end of the pre-reproduction period and the initial stage of the incubation period (PR4, $\mathrm{PR} 5$, and IN1), the estrogen concentrations were significantly higher than those in the other PR stages $(\mathrm{P}<0.05)$. The highest concentration of estrogen was $165 \%$ higher than those in the other stages. However, the testosterone concentration in the early stage of the reproduction period (PR1) was higher than that in the other stages, whereas the progesterone concentration in the early stage of incubation was higher than that in later stages, although the differences were not significant.

The higher level of estrogen at the end stage of the pre-reproduction (PR) period and the early stage of the incubation period (IN) indicates that there was a spike in estrogen production before ovulation in the reproduction cycle. This could be because estrogen can enhance ovum oogenesis (Ma \& Yang, 1999; Peh, Wu, \& 
Lin, 2003). In addition, estrogen can influence calcium metabolism for eggshell formation and promote ovoalbumin, conalbumin, ovomucoid, and lysozyme synthesis (Palmiter, 1972). These changes in estrogen concentration are similar to those observed in black kites (Blas et al., 2010). They could also indicate a cyclical variation in the production of steroid hormones in the avian reproductive cycle (Wingfield \& Famer, 1978; Silverin et al., 1986; Morton, Peterson, Burns, \& Allan, 1990). In the early stages of the pre-reproduction period (particularly in PR1 of the incomplete cycle and PR1-2 of the complete cycle), the testosterone level was higher than that in the other stages, which can probably be ascribed to the heightened territorial behavior of male cockatiel on introduction to a new environment. This phenomenon has also been observed in blackbirds, eider ducks, and song sparrows (Gorman, 1997; Harding \& Follett, 1979; Wingfield, 1984). A higher level of testosterone is related to spermatogenesis (Hirschenhauser, Mostl, \& Kotrschal, 1999) and mating displays in male birds (Cherel, Mauget, Lacroix, \& Gilles, 1994; Saint, Williams, Mickaelian, \& Paillat, 1996).

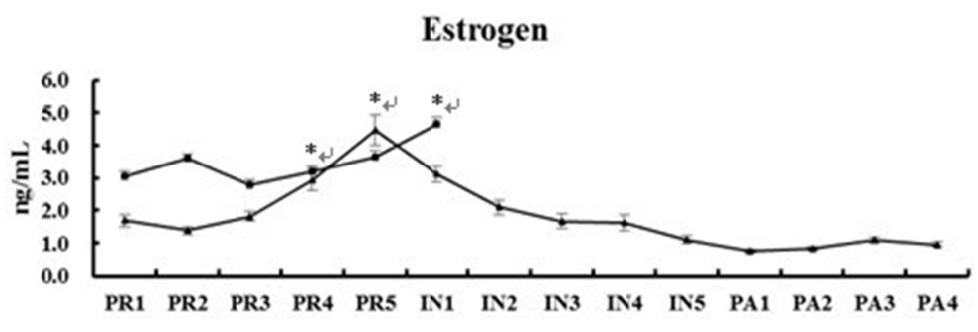

Testosterone

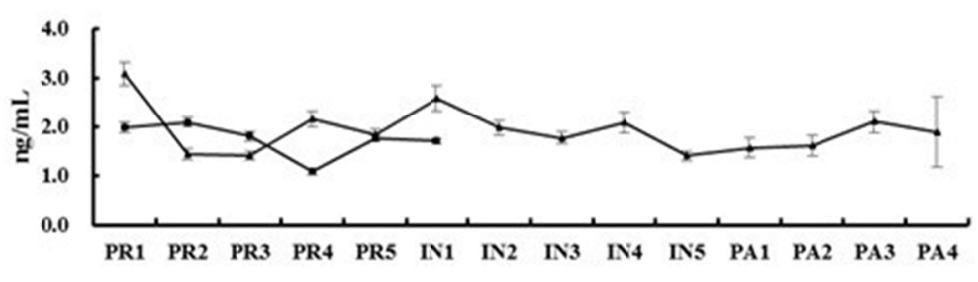

Progesterone

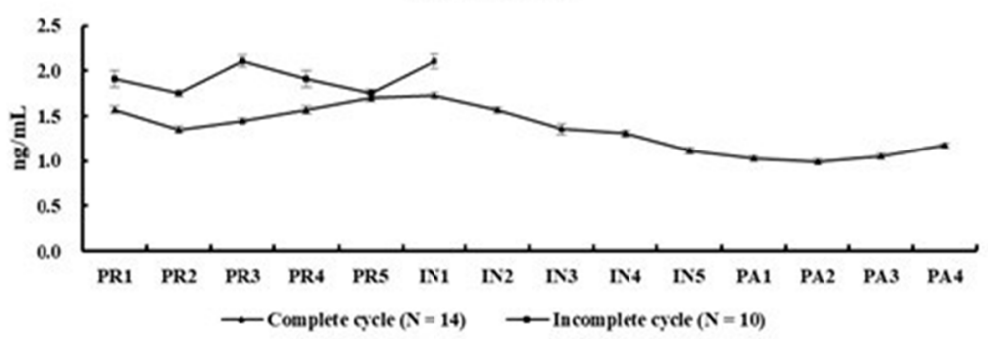

Figure 1. Mean fecal steroid hormone concentrations (mean \pm SD) in cockatiel pairs during different periods of the reproductive cycle. The graphs represent the means of fecal estrogen, testosterone, and progesterone levels of the cockatiel pairs, respectively. The Y-axis represents hormone concentrations, whereas the X-axis represents

the periods of reproduction. PR: the pre-reproduction period, PR1-5: the stages of the PR period. IN: the incubation period, IN1-5: the stages of the IN period. PA: the parental period, PA1-4: the stages of the PA period. Asterisks indicate a significant difference $(\mathrm{P}<0.05)$

The higher progesterone concentrations in the early stage of the incubation period can be attributed to the fact that this hormone can regulate ovulation in avian species (Scanes, 2003). A comparable peak has also been detected in the reproduction cycles of Pycnonotus sinensis (Dawson, 1983) and doves (Silver et al., 1947), indicating that progesterone, as well as the other hormones examined in cockatiels, are similarly regulated in multiple avian species.

\subsection{Nest Boxes Affect Reproductive Hormone Levels}

The levels of steroid hormones during the reproductive cycle of paired cockatiels subjected to the nest box treatment are shown in Figure 2. We found that there were no significant differences in the steroid hormone levels of pairs that had complete and incomplete reproductive cycles. Subsequent to the nest box treatment (NB 
group), estrogen levels fluctuated to a greater extent in the early stages of the pre-reproduction cycle. The males received female stimulation for their mating behavior (Runfeldt \& Wingfield, 1985), and therefore, the testosterone levels in the NB group males were significantly higher during the period of nest box removal (RN) than during other periods $(\mathrm{P}<0.05)$. Elevated testosterone levels have also been observed in the males of macaroni (Eudyptes chrysolophus) and gentoo (Pygoscelis papua) penguins during nesting and mating periods (Williams, 1992). During the RN period of the present study, there were no nest boxes in which the female cockatiels could hide, and this may have led to more frequent couplings with male cockatiels. Mate coupling could be used to evaluate the testosterone levels in male birds and allow for more frequent observations of mating behavior. Indeed, we found that the testosterone levels recorded during the period of nest box removal were higher than those at other periods, particularly in the middle and late stages (Fig. 2, RN2-4). The estrogen level was significantly higher at the end of the reproduction and early incubation periods $(\mathrm{P}<0.05)$, indicating that there was a sexual stimulation under the no-nest box $(\mathrm{CON})$ and nest box $(\mathrm{NB})$ conditions in cockatiels with reproductive experience, as has been observed in budgerigars (Brockway, 1962; 1964; 1965) and canaries (Hinde, 1965; Hinde \& Steel, 1978). These studies on budgerigars and canaries indicate that the provision of nest boxes could induce ovarian development, ovulation, and incubation, thereby explaining the changes in estrogen levels. Although the observed changes in progesterone levels were not significant, we observed that the levels of this hormone did peak at the same time as those of estrogen. This observation is consistent with the findings of Johnson (1990), who showed that the estrogen and progesterone levels in hens peaked at $4 \mathrm{~h}$ before ovulation. In the present study, we found that estrogen levels in cockatiels were lower during the incubation period, and in this regard, it has previously been found that a medial concentration of estrogen is associated with enhanced parental behavior (Cherel et al., 1994; Silver \& Cooper, 1993; Hector, Croxall, \& Follet, 1996), such as feeding, warming, and taking care of nestlings.

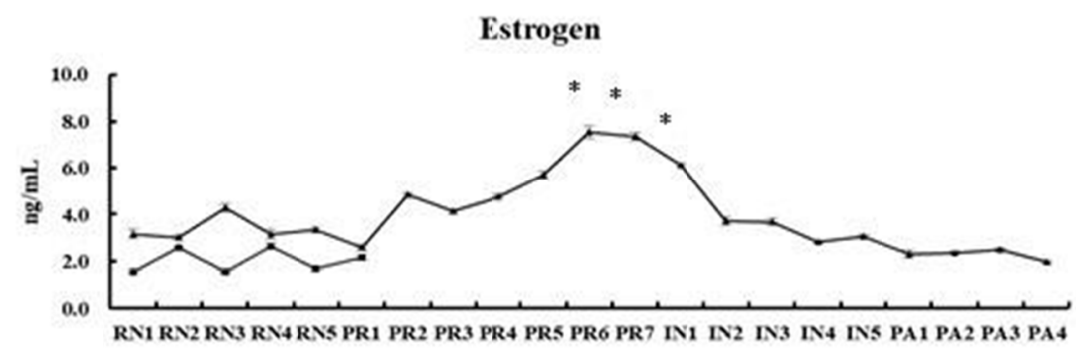

Testosterone
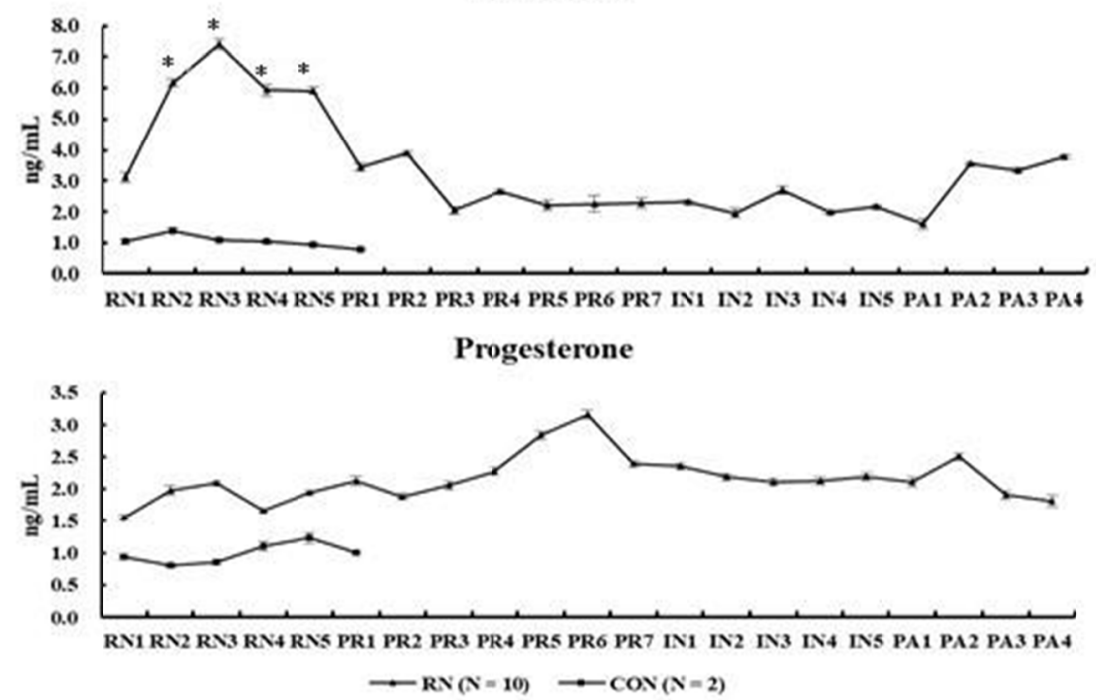

Figure 2. The mean fecal steroid hormone concentrations (mean $\pm \mathrm{SD}$ ) in cockatiel pairs during the different reproductive periods in the nest box treatments. The graphs represent the means of fecal estrogen, testosterone, and progesterone levels of the cockatiel pairs, respectively. The Y-axis represents hormone concentrations, whereas the X-axis represents the periods of reproduction. PR: the pre-reproduction period, PR1-5: the stages of the PR period. IN: the incubation period, IN1-5: the stages of the IN period. PA: the parental period, PA1-4: the stages of the PA period. Asterisks indicate a significant difference $(\mathrm{P}<0.05)$ 
Paired cockatiels generally require a transition period of 30-40 days after completing one reproductive cycle before entering the next reproductive cycle. However, when nest boxes were removed for a time before being replaced, we found that some cockatiels required a transition period of only 6-13 days prior to entering the next cycle, although we did not observe this pattern in all mating pairs. Previous research suggests that reproductively experienced cockatiels typically begin laying eggs within 21 days after nest box presentation (Shields et al., 1989); however, the responses of our cockatiels may have been influenced by individual differences in reproductive and environmental conditions.

\section{Conclusions}

Monitoring of fecal steroid hormones is a non-invasive and valid tool for assessing cockatiel gonadal hormone, and for determining how avian hormones change during reproduction. Concentrations of estrogen undergo significant changes throughout the reproductive cycle and can be used to track variations in the reproductive cycle of cockatiels, such as during the pre-reproduction, incubation, and parental periods. The temporary removal of nest boxes provides a positive stimulus for cockatiel reproduction. In the no-nest box condition, reproductively experienced cockatiels were able to regulate their physical state to enter the next reproductive cycle. The results of nest box re-setting indicate that cockatiels can shorten the interval between two reproductive cycles and could enter the next cycle sooner than those that do not breed seasonally.

\section{References}

Appleby, M. C., \& McRae, H. E. (1986). The individual nest box as a superstimulus for domestic hens. Applied Animal Behaviour Science, 15(2), 169-176. https://doi.org/10.1016/0168-1591(86)90062-6

Blas, J., L. Lopez, A. Tanferna, F. S., \& Hiraldo, F. (2010). Reproductive endocrinology of wild, long-lived raptors. General and Comparative Endocrinology, 168(1), 22-28. https://doi.org/10.1016/j.ygcen.2010.0 3.020

Brockway, B. F. (1962). The effects of nest-entrance positions and male vocalizations on reproduction in budgerigars. Living Bird, 1, 93-101.

Brockway, B. F. (1964). Ethological studies of the budgerigar: Reproductive behavior. Behaviour, 23, 294-323. https://doi.org/10.1163/156853964X00193

Brockway, B. F. (1965). Stimulation of ovarian development and egg laying by male courtship vocalization in budgerigars (Melopsittacus undulatus). Animal Behaviour, 13(4), 575-578. https://doi.org/10.1016/ 0003-3472(65)90123-5

Clark, L., \& Mason, J. R. (1985). Use of nest material as insecticidal and anti-pathogenic agents by the European Starling. Oecologia, 67, 169-176. https://doi.org/10.1007/BF00384280

Cherel, Y., R. Mauget., A. Lacroix, \& Gilles, J. (1994). Seasonal and fasting-related changes in circulating gonadal steroids and prolactin in king penguins, Aptenodyptes patagonicus. Physiological Zoology, 67(5), 1154-1173. https://doi.org/10.1086/physzool.67.5.30163887

Dawson, A. (1983). Plasma gonadal steroid levels in wild starlings (Sturnus vulgaris) during the annual cycle and in relation to the stages of breeding. General and Comparative Endocrinology, 49(2), 286-294. https://doi.org/10.1016/0016-6480(83)90146-6

Forshaw, J. M., \& Cooper, W. T. (1981). Australian Parrots (p. 312). Landsdowne Press, Melbourne, Australia.

Gorman, M. L. (1997). Sexual behavior and plasma androgen concentrations in the male eider duck (Somateria mollissima). Journal of Reproduction and Fertility, 49(2), 225-230. https://doi.org/10.1530/jrf.0.0490225

Goymann, W., Mostl, E., \& Gwinner, E. (2002). Non-invasive methods to measure androgen metabolites in excrements of European stonechats, Saxicola torquata rubicola. General and Comparative Endocrinology, 129(2), 80-87. https://doi.org/10.1016/S0016-6480(02)00520-8

Goymann, W. (2005). Noninvasive monitoring of hormones in bird droppings: Physiological validation, sampling, extraction, sex differences, and the influence of diet on hormone metabolite levels. Annals of the New York Academy of Sciences, 1046(1), 35-53. https://doi.org/10.1196/annals.1343.005

Harding, C. F., \& Follett, B. K. (1979). Hormone changes triggered by aggression in a natural population of blackbirds. Science, 203(4383), 918-920. https://doi.org/10.1126/science.570304

Hector, J. A. L., Croxall, J. P., \& Follet, B. K. (1996). Reproductive endocrinology of the wandering albatross Diomedea exulans in relation to biennial breeding and deferred sexual maturity. Ibis, 128, 9-22. https://doi.org/10.1111/j.1474-919X.1986.tb02088.x 
Hinde, R. A. (1965). Interaction of internal and external factors in integration of canary reproduction. In F. A. Beach (Ed.), Sex and Behavior (pp. 381-415). Wiley, New York.

Hinde, R. A., \& Steel, E. (1978). The influence of day length and male vocalizations on the estrogen-dependent behavior of female canaries and budgerigars, with discussion of data from other species. In J. S. Rosenblatt, R. A. Hinde, E. Shaw, \& C. Beer (Eds.), Advances in the study of behavior (pp. 39-73). Academic Press, New York.

Hirschenhauser, K., Mostl, E., \& Kotrschal, K. (1999). Seasonal patterns of sex steroids determined from feces in different social categories of Greylag geese (Anser anser). General and Comparative Endocrinology, 114(1), 67-79. https://doi.org/10.1006/gcen.1998.7236

Hutchison, R. E. (1974). Temporal patterning of external stimuli and reproductive behavior in female budgerigars. Animal Behaviour, 22(1), 150-157. https://doi.org/10.1016/S0003-3472(74)80064-3

Johnson, A. L. (1990). Steroidogenesis and actions of steroids in the hen ovary. Critical Reviews in Poultry Biology, 2, 319-346.

Khan, M. Z., Altmann, J., Isani, S. S., \& Yu, J. (2002). A matter of time: Evaluating the storage of fecal samples for steroid analysis. General and Comparative Endocrinology, 128(1), 57-64. https://doi.org/10.1016/ S0016-6480(02)00063-1

Kotrschal, K., Dittami, J., Hirschenhauser, K., Moestl, E., \& Peczely, P. (2000). Effects of physiological and social challenges in different seasons on fecal testosterone and corticosterone in male domestic geese. Acta Ethologica, 2(2), 115-122. https://doi.org/10.1007/s102110000016

Lin, P.-Y. (2008). The effect of transfer stress on fecal corticosterone concentration in Collared Scops Owls (Master Diss. National Chung Hsing University, Taichung, Taiwan).

Ma, C. X., \& Yang, X. K. (1999). Essential Reproduction (4th ed.). National Institute for Compilation and Translation, Taipei City. Taiwan.

Martin, S. G., \& Millam, J. R. (1995). Nest box selection by floor laying and reproductively naïve captive cockatiels (Nymphicus hollandicus). Applied Animal Behaviour Science, 43(2), 95-109. https://doi.org/ 10.1016/0168-1591(95)00559-B

Morton, M. L., Peterson, L. E., Burns, D. M., \& Allan, N. (1990). Seasonal and age-related changes in plasma testosterone levels in mountain white-crowned sparrows. The Condor: Ornithological Applications, 92(1), 166-173. https://doi.org/10.2307/1368395

Palmiter, R. D. (1972). Regulation of protein synthesis in chick oviduct. I. Independent regulation of ovalbumin, conalbumin, ovomucoid, and lysozyme induction. Journal of Biological Chemistry, 247, 6450-6461.

Peh, H. C., Wu, L. S., \& Lin, J. H. (2003). Endocrinology of Domestic Animal. Yi-Hsien Publishing CO., Ltd. Taipei City, Taiwan.

Putnam, R. J., \& Hinde, R. A. (1973). Effects of light regime and breeding experience on budgerigar reproduction. Journal of Zoology, 170, 475-483. https://doi.org/10.1111/j.1469-7998.1973.tb05061.x

Runfeldt, S., \& Wingfield, J. C. (1985). Experimentally prolonged sexual activity in female sparrows delays termination of reproductive activity in their untreated mates. Animal Behaviour, 33(2), 403-410. https://doi.org/10.1016/S0003-3472(85)80064-6

Saint, J. M., Williams, J., Mickaelian, I., \& Paillat, P. (1996). Seasonal variation of LH, sex steroids, body mass, molt, display, and laying in two subspecies of Houbara bustard, Chlamydotis undulata macqueenii and Chlamydotis undulata undulata, housed in outdoor cages under natural conditions. General and Comparative Endocrinology, 102(1), 102-112. https://doi.org/10.1006/gcen.1996.0051

Scanes, C. G. (2003). Sturkie's Avian Physiology (6th ed.). Academic Press, Elsevier.

Schumacher, M., \& Balthazart, J. (1983). The effects of testosterone and its metabolites on sexual behavior and morphology in male and female Japanese quail. Physiology \& Behavior, 30(3), 335-339. https://doi.org/ 10.1016/0031-9384(83)90135-X

Shields, K. M., Yamamoto, J. T., \& Millam, J. R. (1989). Reproductive behavior and LH levels of cockatiels (Nymphicus hollandicus) associated with photostimulation, nest-box presentation, and degree of mate access. Hormones and Behavior, 23(1), 68-82. https://doi.org/10.1016/0018-506X(89)90075-5 
Silver, R., Reboulleau, C., Lehrman, D. S., \& Feder, H. H. (1974). Radioimmunoassay of plasma progesterone during the reproductive cycle of male and female ring doves (Streptopelia risoria). Endocrinology, 94(6), 1547-1554. https://doi.org/10.1210/endo-94-6-1547

Silverin, B., Viebke, P. A., \& Westin, J. (1986). Seasonal changes in plasma levels of LH and gonadal steroids in free-living willow tits, Parus montanus. Ornis Scandinavica, 17(3), 230-236. https://doi.org/10.2307/ 3676831

Staley, A. M., Blanco, J. M., Jr. Dufty, A. M., Wildt, D. E., \& Monfort, S. L. (2007). Fecal steroid monitoring for assessing gonadal and adrenal activity in the golden eagle and peregrine falcon. Journal of Comparative Physiology B, 177(6), 609-622. https://doi.org/10.1007/s00360-007-0159-2

Silver, R., \& Cooper, M. (1993). Avian behavioral endocrinology. Bioscience, 33, 567-572. https://doi.org/ $10.2307 / 1309207$

Wasser, S. K., Monfort, S. L., Southers, J., \& Wildt, D. E. (1994). Excretion rates and metabolites of oestradiol and progesterone in baboon (Papio cynocephalus cynocephalus) faeces. Journal of Reproduction and Fertility, 101, 213-220. https://doi.org/10.1530/jrf.0.1010213

Whitten, P. L., Brockman, D. K., \& Stavisky, R. C. (1998). Recent advances in non-invasive techniques to monitor hormone-behavior interactions. American Journal of Physical Anthropology, 41, 1-23. https://doi.org/10.1002/(SICI)1096-8644(1998)107:27+\%3C1::AID-AJPA2\%3E3.0.CO;2-H

Wingfield, J. C., \& Famer, D. S. (1978). The endocrinology of a natural breeding population of the white-crowned sparrow (Zonotrichia leucophrys pugetensis). Physiological Zoology, 51(2), 188-205. https://doi.org/10.1086/physzool.51.2.30157866

Wingfield, J. C. (1984). Environmental and endocrine control of reproduction in the song sparrow, Melospiza melodia. II. Agonistic interactions as environmental information stimulating secretion of testosterone. General and Comparative Endocrinology, 56(3), 417-424. https://doi.org/10.1016/0016-6480(84)90084-4

Williams, T. D. (1992). Reproductive endocrinology of macaroni (Eudyptes chrysolophus) and gentoo (Pygoscelis papua) penguins. I. Seasonal changes in plasma levels of gonadal steroids and LH in breeding adults. General and Comparative Endocrinology, 85(2), 230-240. https://doi.org/10.1016/0016-6480(92) 90006-6

\section{Copyrights}

Copyright for this article is retained by the author(s), with first publication rights granted to the journal.

This is an open-access article distributed under the terms and conditions of the Creative Commons Attribution license (http://creativecommons.org/licenses/by/4.0/). 